\title{
Chapter 1 \\ Machine-Learning Defined Networking: Towards Intelligent Networks
}

\author{
Sebastian Troia
}

\subsection{Introduction}

On October 29, 1969, the world was changed forever. At $10: 30$ p.m., a student programmer at the University of California at Los Angeles (UCLA) named Charley Kline sent the letter "l" and the letter "o" electronically more than 350 miles to a Stanford Research Institute computer in Menlo Park, California. In that specific moment the Internet history was made and a technological revolution had begun. 26 years after Charley Kline's first message, the number of people interconnected by Internet was 16 million and in just 50 years it reached 4 billion $(58.8 \%$ of world population). ${ }^{1}$ Bit streams of information over the Internet Protocol (IP), known as IP traffic, continuously crosses the world to reach: end users through High Definition (HD) screens (either in mobile and fixed devices), connected machines (Internet of Things), and IT infrastructures, i.e., public or private datacenters and the Cloud infrastructures. IP traffic is powered by ubiquitous Internet connectivity provided by CSPs. Every day, around 18 billion Internet-connected devices and 4 billion global Internet users transfer 900 Petabytes $\left(10^{15}\right.$ bytes) of IP traffic across the globe [1]; by the end of 2021, the global IP traffic will reach 3.3 zettabytes $\left(10^{21}\right.$ bytes) [2]. Moreover, with the advent of $5 \mathrm{G}$ technology, we are witnessing the development of increasingly bandwidth-hungry network applications, such as enhanced mobile broadband, massive machine-type communications and ultra-reliable low-latency communications. Contrary to the evolution of previous generations, $5 \mathrm{G}$ will require not only improved networking solutions but also more sophisticated mechanisms for traffic management to fulfill stronger end-to-end Quality of Service (QoS) require-

\footnotetext{
${ }^{1}$ Online: https://www.internetworldstats.com/stats.htm.

\section{S. Troia $(\bowtie)$}

Dipartimento di Elettronica, Informazione e Bioingegneria, Politecnico di Milano,

Via Giuseppe Ponzio, 34, 20133 Milano, MI, Italy

e-mail: sebastian.troia@polimi.it 
ments of numerous different "verticals", such as automotive, manufacturing, energy, eHealth, agriculture, etc.

Traditional network architectures are ill-suited to meet the requirements of the aforementioned emerging Internet services. The common practice in CSP networks is to perform a static resource allocation which meets the peak-hour traffic demand. This method leads to poor network and energy efficiency, as outside the peak hour resources will be over-provisioned. As a consequence, the Internet has become one of the major energy consumer in the world. This situation is forcing the CSPs to change the underlying network technologies, and have started to look at new technological solutions that increase the level of programmability, control, and flexibility of configuration, while reducing the overall costs related to network operations.

This Ph.D. thesis [3] focuses on three of the major disruptive technologies in networking in the last two decades: Software Defined Networking (SDN), Network Function Virtualization (NFV) and Machine Learning (ML).

SDN is one of the fastest growing markets in Information and Communication Technologies (ICT), from 289 million in 2015 to 8:7 billion in 2020, with a Compound Annual Growth Rate (CAGR) of $98 \%$ [4]. It combines automation, agility and development of applications to deliver network services in a deterministic, dynamic, and scalable manner. By separation of control and data plane, SDN brings networking back to centralized control, allowing applications to program the network forwarding rules through logically centralized controllers. SDN brings a promising solution to introduce network programmability and accelerate networking innovation. For instance, network programmability allows to implement intelligent dynamic optimization methods to improve performance and energy efficiency. The application of SDN in Enterprise Networking (EN) is called Software Defined Wide Area Network (SD-WAN) [5] and represents an emerging paradigm that introduces the advantages of SDN into EN. SD-WAN is a revolutionary way to simplify enterprise networks and achieve optimal application performance using centrally managed WAN virtualization. Unlike traditional WAN connections, SD-WAN provides high network agility and cost savings.

By leveraging virtualization technologies, the European Telecommunications Standards Institute (ETSI) proposed NFV to virtualize network services previously performed by dedicated proprietary hardware. NFV is the paradigm of transferring network functions from dedicated hardware appliances to software-based applications running on commercial off-the-shelf equipment [6]. Network devices are no longer proprietary but are open to host software from different vendors, drastically reducing CapEx and OpEx. Although the adoption of SDN and NFV in networking brings revolutionary benefits in scale and agility, it also brings a whole new level of complexity. Virtualization breaks traditional networking into dynamic components and layers that have to work in unison and that can change at any given time [7], for instance, a virtualized firewall can be subject to continuous updates by the CSP. The high complexity introduced by these new technologies has led research to focus on increasingly intelligent methods and algorithms to optimize the allocation of resources in all network segments: access, metro and core. In recent years, Machine Learning has become the main mathematical tool used by researchers in this 
field. ML is a sub-field of computer science that originated from the study of pattern recognition and computational learning theory in artificial intelligence. By leveraging complex mathematical and statistical tools, computers can learn by input data how to solve specific problems that have been traditionally solved by human beings [8]. ML explores algorithms that can "learn" from and make predictions out of input data. The learning phase is also referred to as "training" an algorithm, by iterative and feedback-based mechanisms, to solve specific problems. Such algorithms operate by building a model in order to make data-driven predictions or decisions, rather than following static program instructions. ML algorithms are typically classified into three different categories:

- Supervised Learning (SL) is used in a variety of applications, such as speech recognition, spam detection and object recognition. The goal is to predict the value of one or more output variables given the value of a vector of input variables. The output variable can be a continuous variable (regression problem) or a discrete variable (classification problem). A training data set comprises a set of samples of the input variables and the corresponding output values.

- Unsupervised Learning (UL) consists of training an algorithm using only input vector data with the aim to find hidden patterns and similarities. Social network analysis, genes clustering and market research are among the most successful applications of unsupervised learning methods. It can address different tasks, such as clustering or cluster analysis. Clustering is the process of grouping data so that the intra-cluster similarity among the input data samples is high, while the inter-cluster similarity is low.

- Reinforcement Learning (RL) is an approach to ML that trains algorithms using a system of positive and negative rewards. Contrary to what happens with the supervised and unsupervised learning, in RL we do not have training, validation and test set of data defined "a priori". An RL algorithm, or agent, learns how to perform a task by interacting with its environment. The interaction is defined in terms of specific actions, observations and rewards.

This chapter presents the main results of my $\mathrm{PhD}$ thesis [3] by introducing novel ML-based algorithms to solve various network optimization problems. The thesis provides contributions to all the ML branches by implementing supervised, unsupervised and reinforcement learning algorithms together with their implementations in real and emulated SDN/NFV testbed scenarios.

\subsection{Network Traffic Prediction}

Mobile, metro and core networks often suffer from resource inefficiency due to overprovisioning. It's a common practice to perform static resource allocation based on the peak-hour demand, because the current operational processes used by the network operators are too slow to dynamically allocate the resources following the daily demand variations. Over-provisioning leads to poor energy efficiency and high OpEx, 
as the resources are sub-utilized outside of the peak hour. Moreover, as the peakhour to average demand ratio continue to increase [2], the static resource allocation lead to higher and unnecessary OpEx and CapEx. As such, understanding how to correctly predict network behaviour plays a vital role in the management and provisioning of mobile and fixed network services. Network traffic prediction has become very important for network providers. Having an accurate traffic prediction tool is essential for most network management tasks, such as resource allocation, shorttime traffic scheduling or re-routing, long-term capacity planning, network design and network anomaly detection. A proactive prediction-based approach allows network providers to optimize network resource allocation, potentially improving the QoS. With this aim, we introduce a matheuristic (i.e. interoperation of heuristic and mathematical programming) for dynamic network optimization. In Fig. 1.1 we show the proposed model. Traffic is predicted on different spatial locations developing different machine-learning algorithms, such as Artificial Neural Network (ANN) [9], Recurrent Neural Network (RNN) [10] and Diffusion Convolutional RNN (DCRNN) [11]. The predicted traffic-demand is then used to optimize the network at various hours during the day, so to adapt resource occupation to the actual traffic volume. The optimization problem solvers are based on Integer Linear Programming (ILP) and heuristic algorithms; their goal is to minimize the power and energy consumption of the network elements, providing weights to assign to network links every hour. After that, a weighted graph is generated to route the actual traffic demands by a minimum-cost path algorithm. The time required by optimization is not an issue, since prediction allows to start performing optimization computation in advance (Offline). Such feature makes the solution reported suitable to be implemented as an SDN application for $5 \mathrm{G}$ scenarios. We have chosen the metro infrastructure of a mobile operator as use case; in particular, the objective of the resource optimization is the optical metro network used as the backbone for the mobile service. The tests

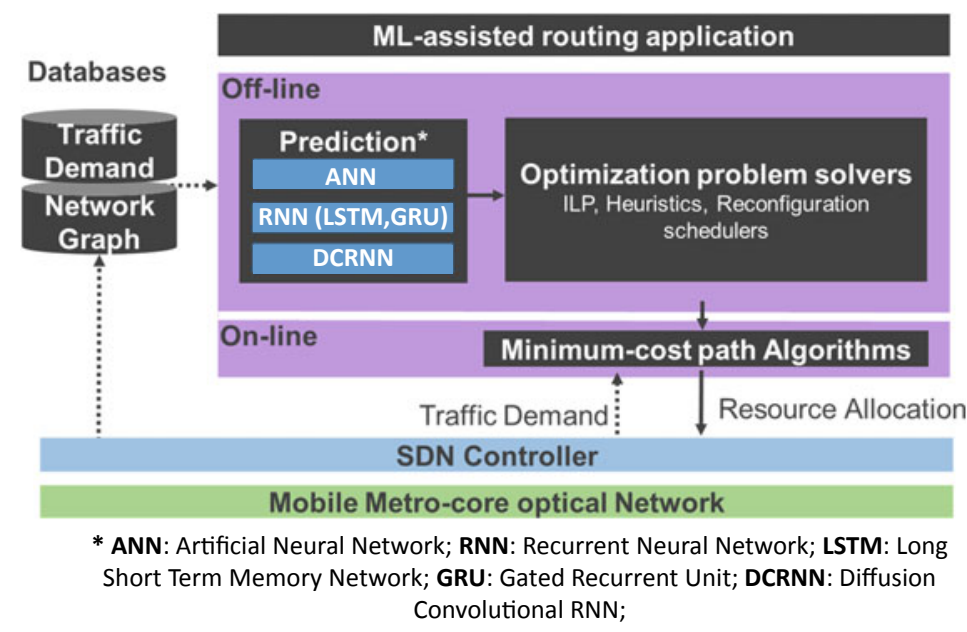

Fig. 1.1 Dynamic optical routing matheuristic for software-defined mobile metro network [9] 
demonstrate the effectiveness of our methodology, with results that match almost perfectly th!htbe behaviour of a network that performs optical routing reconfiguration with a perfect, oracle-like traffic prediction. The methodology and the detailed results can be found in Chaps. 2 and 3 of my Ph.D. thesis and in the following papers: [9-12].

\subsection{Network Traffic Pattern Identification}

Due to the highly predictable daily movements of citizens in urban areas, mobile traffic shows repetitive patterns with spatio-temporal variations. This phenomenon is known as tidal effect analogy to the rise and fall of the sea levels. Recognizing and defining traffic load patterns at the base station thus plays a vital role in traffic engineering, network design and load balancing since it represents an important solution for the Internet Service Providers (ISPs) that face network congestion problems or over-provisioning of the link capacity. State of the art works have dealt with the classification and identification of patterns through the use of techniques, which inspect the flow of data of a particular application. In the previous section we have shown dynamic network optimization techniques based on supervised learning algorithms, or prediction algorithms, while in this section, we use unsupervised learning to "learn" periodic trends (or patterns) from traffic data over time. We wanted to answer to the following questions: Do different areas of the city with the same social function display the same typical traffic pattern? Can they be used to optimize the metro-core optical network? In order to answer to these questions we analysed datasets concerning the city of Milan [13, 14]. They show information regarding the internet traffic of Telecom Italia (TIM) service provider and the point of interests located throughout the city, such as schools, restaurants, social services, etc., that reveal the social function of a particular area. We developed a novel model for basic pattern identification based on matrix factorization methods by integrating the aforementioned datasets in order to assess the similarity of traffic patterns related to areas with the same type of point of interests. In the field of pattern recognition, the Non-negative Matrix Factorization (NMF) is one of the most used method thanks to the ability to detect basic flows inside large matrices. First, we apply the classical NMF method to a real-world dataset that collects the data traffic of mobile users at the base station level in the city of Milan. Afterwords, we propose an integration of multi-domain data on the basis of NMF and denote it as Collective-NMF based model. The final results show the presence of very similar patterns located in distant areas of the city that share the same point of interests, such as schools, public parks, commuting areas, clubs, commercial areas (see Fig. 1.2). The experiments confirm the presence of regular and periodic traffic due to the tidal effect experienced by the network. In fact, it depends not only on user habits but also by the land usage and POIs scattered in the city area. Taking advantage of the mobile traffic patterns, we propose two mathematical models with the goal of predicting how and when to optimize the resource allocation in the underlying physical network. The methodology and the detailed results can be found in Chaps. 4 and 5 of the Ph.D thesis and in the following papers: [15-17]. 

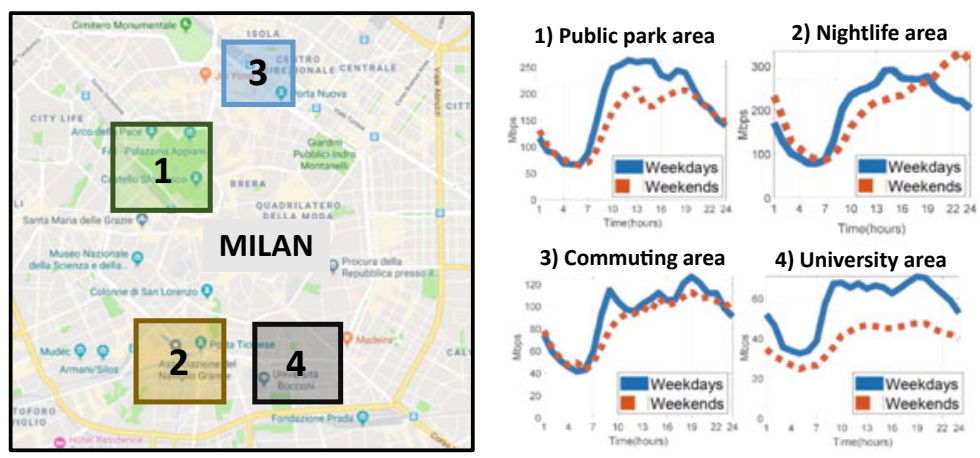

Fig. 1.2 Typical mobile traffic patterns in the city of Milan [15]

\subsection{Reinforcement Learning for Adaptive Network Resource Allocation}

While in the previous sections we investigated machine learning algorithms for network optimization based on supervised and unsupervised learning, in this section, we investigate the application of RL for performing dynamic Service Function Chain (SFC) resources allocation in SDN/NFV enabled metro-core optical networks. RL allows to build a self-learning system able to solve highly complex problems by employing RL agents to learn policies from an evolving network environment. Specifically, we build an RL system able to optimize the resources allocation of SFCs in a multi-layer network (packet over flexi-grid optical layer). An SFC is composed of an ordered sequence of Virtual Network Functions (VNFs). Therefore, given a set of SFC provisioning requests and their QoS requirements, the proposed method finds the optimal positioning of the VNFs in the service layer and allocates the network resources of the IP/MPLS and optical layer, respectively. Furthermore, in order to meet the SFC traffic variation over time, it dynamically predicts if and when to reconfigure the SFCs that no longer meet the required QoS criteria. Each reconfiguration implies an availability penalty on the service as it requires a temporary service interruption to allow for VNF migration and/or network resource reallocation. Thus, the mechanism presented here can be seen as a way of spending reconfiguration tokens over the span of a day. The proposed RL-based algorithm aims to spend these tokens in order to minimize the blocking probability of traffic requests finding the best action in the trade-off between availability and performance. Reinforcement learning is an approach to machine learning that trains algorithms using a system of positive and negative rewards. An RL algorithm, or agent, learns how to perform a task by interacting with its environment. The interaction is defined in terms of specific actions, observations and rewards. As we can see from Fig. 1.3, an agent performs an action at time $\mathrm{t}$ based on the reward and state (or observations) obtained by the environment. The action performed by the 
Fig. 1.3 Schema of the proposed RL system [18]

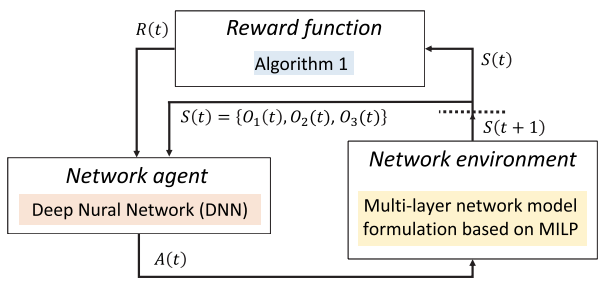

agent will produce another state of the environment and a reward at a time $t+1$ and so on.

The environment, namely network environment, consists of a multi-layer model formulation based on Mixed ILP (MILP) that, given a set of SFC requests, finds the optimal VNF placement and Routing and Wavelength Assignment (RWA). The objective function of the MILP aims to maximize the number of successfully routed SFCs, minimizing: reconfiguration penalty, blocking probability and power consumption of network elements. At each time step t, i.e. hour, the network environment exposes its state $\mathrm{S}(\mathrm{t})$ made by 3 observations: $O_{1}(t)$ : total number of reconfigurations of each SFC, at time t; $O_{2}(t)$ : total number of blocked requests of each SFC, at time $\mathrm{t} ; \mathrm{O}_{3}(t)$ : traffic volume request in Gbps of each SFC, at time t. As shown in Fig. 1.3, the agent, namely Network Agent, is a Dense Neural Network (DNN) that takes as input the state of the environment $S(t)$ and the result of the reward function $R(t)$. As output, the agent decides if one or more SFCs need to be reconfigured. This decision is performed by means of an action $\mathrm{A}(\mathrm{t})$ executed on the environment. The reward function represents the effect of the action performed by the agent. It depends on the number of blocked service-chain requests; $\mathrm{R}(\mathrm{t})$ is positive if the agent managed to reconfigure the network in order not to have blocked requests, otherwise it is negative. Results show that, initially the system assigns negative rewards that decreases hour by hour when the algorithm does not block the requests. After few days of training, the RL agent figured out how to maximize the reward function and keep the objective function of the MILP stable to a minimum value respect to the case in which we do not use RL. In other words, it learnt how and when to reconfigure the SFCs in order to route the traffic requests by decreasing the blocking probability. The methodology and the detailed results can be found in Chap. 6 of the Ph.D. thesis and in the following paper: [18].

\subsection{Implementation of Machine Learning in Real SDN/NFV Testbeds}

While in the previous sections we have proposed novel algorithms in simulated networks, in this section we introduce briefly three direct applications of ML-based algorithms on different emulated and real SDN/NFV testbed scenarios. 
1. First, we developed the network planner module under the Metro-Haul European project ${ }^{2}$ in which ML-based algorithms interact with the control plane of the proposed SDN/NFV infrastructure. We introduced the software components inside the planning tool, which compose a framework that enables intelligent optimization algorithms based on ML to assist the control plane in taking strategic decisions. We presented a demonstration of a ML-assisted routing algorithm in an emulated network scenario. The proposed framework aims at guaranteeing a fair behavior towards past, current and future requests as network resource allocation decisions are assisted with ML approaches. The detailed results can be found in Chap. 7 of the Ph.D. thesis and in the following papers: [19-22].

2. Then, we developed an experimental ML-enabled SDN/NFV service orchestrator called SENATUS, targeting research environment, as devised to support testbeds for the development and validation of network services and network planning algorithms. It implements functions for managing SDN networks as well as for deploying network services on NFV infrastructure. SENATUS introduces an interface for horizontal communication that allows interworking with experimental ML modules and optimization algorithms. In addition, we present two testbed scenarios devised for testing the SDN features of the service orchestrator. The first is a physical implementation deployed at the BONSAI laboratory of Politecnico di Milano. ${ }^{3}$ This testbed, named Minilab, provides both SDN and NVF capabilities, taking full use of SENATUS. The second testbed is an IP-over-WDM ${ }^{4}$ network and it has been developed in collaboration with the Scuola Superiore Sant'Anna (Pisa, Italy). The testbed is composed by an IP network over an optical network infrastructure. The detailed results can be found in Chap. 8 of the Ph.D. thesis and in the following papers: [23-25].

3. As last, we present an SD-WAN open-source solution that can be tested in real network scenarios. We have implemented active and passive monitoring methods and developed classical and RL-based algorithms to improve the network performance of enterprise networks. We present two testbeds: 1) The first one is a lab implementation of SD-WAN based on open source components such as OpenDaylight SDN controller and OpenvSwitches; 2) The second is a field deployment of such solution in a real network located in an Italian city. These testbeds target the monitoring and the traffic engineering features based on RL of an SD-WAN solution and explores different approaches to understand their advantages and limitations. Our implementations provide an overlay WAN with controlled performance in terms of delay, losses and jitter over low-cost public Internet connectivity. Detailed results can be found in Chap. 9 of the Ph.D. thesis and in the following paper: [26].

\footnotetext{
${ }^{2}$ Online: https://metro-haul.eu/.

${ }^{3}$ Online: https://www.bonsai.deib.polimi.it/.

${ }^{4}$ Wavelength Division Multiplexing (WDM).
} 


\subsection{Concluding Remarks}

Machine learning is one of the game-changing technologies which only recently has become mature. With the growing availability of data, computing power and platforms, ML is now applicable within the academic world and by large companies. It is an extremely powerful technique which is able to perform intelligent tasks which were up to now usually done by humans. The networking community welcomed ML as the main mathematical tool for solving complicated optimization problems. ML can recognize, predict, advice, optimize and classify, and therefore it can support the automation of various network operations enhancing the telecom industry. This PhD research work introduces the concept of Machine-Learning defined Networking. It is an approach to network management, based on SDN and NFV, that enables self-learning algorithms to improve network performance and monitoring. We have demonstrated the effectiveness of this approach by developing different algorithms able to predict and classify network traffic in order to optimize $5 \mathrm{G}$ metrocore networks. ML represents an essential asset for building management tools for ever increasing intelligent networks. Research on this topic is growing at a dizzying pace and is opening up research directions for many areas in telecommunications.

Acknowledgements Special thanks go to my Ph.D. supervisor Prof. Guido Maier for his continuous support and guidance.

\section{References}

1. Cisco (2019) Cisco systems. http://www.cisco.com/

2. Cisco Visual Networking Index (2016) Global mobile data traffic forecast update, 2015-2020. http://www.cisco.com/

3. Troia S (2020) Machine-learning defined networking: applications for the $5 \mathrm{G}$ metro-core, $\mathrm{PhD}$ thesis

4. His markit (2019). https://technology.ihs.com/

5. Uppal Sanjay et al (2015) Software defined WAN for dummies. Wiley, West Sussex

6. Hawilo $\mathrm{H}$ et al (2014) Nfv: state of the art, challenges, and implementation in next generation mobile networks (vepc). IEEE Netw 28(6):18-26

7. Yousaf FZ et al (2017) Nfv and sdn key technology enablers for $5 \mathrm{~g}$ networks. IEEE JSAC 35(11):2468-2478

8. Musumeci F et al (2019) An overview on application of machine learning techniques in optical networks. IEEE Commun Surv Tutor 21(2):1383-1408

9. Alvizu R et al (2017) Matheuristic with machine-learning-based prediction for software-defined mobile metro-core networks. IEEE-OSA JOCN 9(9):D19-D30

10. Troia S et al (2018) Deep learning-based traffic prediction for network optimization. In: 20th ICTON, Bucharest, Romania

11. Andreoletti D et al (2019) Network traffic prediction based on diffusion convolutional recurrent neural networks. In: INFOCOM network intelligence workshop, France, Paris

12. Alvizu R et al (2018) Machine-learning-based prediction and optimization of mobile metrocore networks. In: IEEE photonics society summer topical meeting series (SUM), Waikoloa Village, HI, USA

13. TIM (2014) Big data challenge. https://dandelion.eu/datamine/open-big-data/ 
14. Regione Lombardia (2013) Servizi Comunali Milano. http://www.territorio.regione.lombardia. it/

15. Troia $\mathrm{S}$ et al (2017) Identification of tidal-traffic patterns in metro-area mobile networks via Matrix Factorization based model. In: IEEE PerCom workshops, Kona, HI

16. Troia $S$ et al (2019) Dynamic network slicing based on tidal traffic patterns in metro-core optical networks. In: IEEE HPSR, Xi'An, China

17. Troia $S$ et al (2020) Dynamic programming of network slices in software-defined metro-core optical networks. In: OSN, vol 36, pp 100551

18. Troia $S$ et al (2019) Reinforcement learning for service function chain reconfiguration in NFVSDN metro-core optical networks. IEEE Access 7(1):1-14

19. Troia $\mathrm{S}$ et al (2018) Machine-learning-assisted routing in SDN-based optical networks. In: ECOC, Italy, Rome

20. Troia $\mathrm{S}$ et al (2019) Machine learning-assisted planning and provisioning for SDN/NFVenabled metropolitan networks. In: EuCNC, Valencia, Spain

21. Martín I et al (2018) Is machine learning suitable for solving RWA problems in optical networks?. In: ECOC, Italy, Rome

22. Martín I et al (2019) Machine learning-based routing and wavelength assignment in softwaredefined optical networks. IEEE TNSM 16(3):871-883

23. Troia S et al (2018) SENATUS: an experimental SDN/NFV orchestrator. In: IEEE NFV-SDN, Verona, Italy

24. Troia $S$ et al (2019) Dynamic virtual network function placement over a software-defined optical network. In: OFC, San Diego, USA

25. Troia $S$ et al (2019) Portable minilab for hands-on experimentation with software defined networking. In: ConTEL, Graz, Austria

26. Troia $\mathrm{S}$ et al (2020) SD-WAN: an open-source implementation for enterprise networking services. In: 22th ICTON, Bari, Italy

Open Access This chapter is licensed under the terms of the Creative Commons Attribution 4.0 International License (http://creativecommons.org/licenses/by/4.0/), which permits use, sharing, adaptation, distribution and reproduction in any medium or format, as long as you give appropriate credit to the original author(s) and the source, provide a link to the Creative Commons license and indicate if changes were made.

The images or other third party material in this chapter are included in the chapter's Creative Commons license, unless indicated otherwise in a credit line to the material. If material is not included in the chapter's Creative Commons license and your intended use is not permitted by statutory regulation or exceeds the permitted use, you will need to obtain permission directly from the copyright holder. 